\title{
Differential in vivo expression of mycobacterial antigens in Mycobacterium tuberculosis infected lungs and lymph node tissues
}

\author{
Tehmina Mustafa ${ }^{1,2^{*}}$, Nils Anders Leversen ${ }^{3}$, Lisbet Sviland ${ }^{4,5}$ and Harald Gotten Wiker ${ }^{3,6}$
}

\begin{abstract}
Background: The clinical course of tuberculosis (TB) infection, bacterial load and the morphology of lesions vary between pulmonary and extrapulmonary TB. Antigens expressed in abundance during infection could represent relevant antigens in the development of diagnostic tools, but little is known about the in vivo expression of various M. tuberculosis antigens in different clinical manifestations. The aim of this study was to study the differences in the presence of major secreted as well as somatic mycobacterial antigens in host tissues during advanced rapidly progressing and fatal pulmonary disease with mainly pneumonic infiltrates and high bacterial load, and to compare this to the presence of the same antigens in TB lymphadenitis cases, which is mainly chronic and self-limiting disease with organised granulomas and lower bacterial load.

Methods: Human pulmonary $(n=3)$ and lymph node $(n=17)$ TB biopsies, and non-TB controls $(n=12)$ were studied. Ziehl-Neelsen stain, nested PCR 156110 and immunohistochemistry were performed. Major secreted (MPT32, MPT44, MPT46, MPT51, MPT53, MPT59, MPT63, and MPT64) and somatic mycobacterial antigens (Mce1A, Hsp65, and MPT57) were detected by using rabbit polyclonal antibodies.

Results: Plenty of bacilli were detectable with Ziehl-Neelsen stain in the lung biopsies while no bacilli were detected in the lymph node biopsies. All the cases were shown to be positive by PCR. Both secretory and somatic antigens were expressed in abundance in pulmonary infiltrates, while primarily somatic antigens were detected in the lymphadenitis cases. Of the secreted antigens, only MPT64 was consistently detected in both cases, indicating a preferential accumulation of this antigen within the inflammatory cells, even if the cells of the granuloma can efficiently restrict bacterial growth and clear away the secreted antigens.

Conclusions: This study shows that major secreted mycobacterial antigens were found in high amounts in advanced pulmonary lesions without proper granuloma formation, while their level of staining was very low, or absent, in the lymph node TB lesions with organised granulomas and very low bacillary load, with one exception of MPT64, suggesting its role in the persistence of chronic infection. These findings have implication for development of new diagnostic tools.
\end{abstract}

Keywords: Mycobacterium tuberculosis, Intracellular infection, Pathology, Granuloma, MPT32, MPT44, MPT46, MPT51, MPT53, MTP59, MPT63, MPT64, Mce1A, Hsp65, MPT57

\footnotetext{
* Correspondence: tehmina.mustafa@cih.uib.no

${ }^{1}$ Centre for International Health, Department of Global Public Health and

Primary Care, University of Bergen, Bergen, Norway

${ }^{2}$ Department of Thoracic Medicine, Haukeland University Hospital, Bergen,

Norway

Full list of author information is available at the end of the article
} 


\section{Background}

Infection with Mycobacterium tuberculosis represents a major disease burden globally. There are about 8,7 million new cases of tuberculosis (TB) worldwide and at least 1.4 million deaths per year [1]. Extra pulmonary TB accounts for approximately one-fifth of $\mathrm{TB}$ cases among immune-competent individuals and up to one-half in HIV-infected individuals [1-4]. Though any organ in the body can be involved, lymph nodes are the most common form of extra-pulmonary TB in both adults and children [5,6]. Immune responses in TB differ between various disease sites and in various forms of disease [7,8]. TB lymphadenitis is typically a self-contained, while pulmonary $\mathrm{TB}$ is usually a rapidly progressive and fatal disease without adequate chemotherapy.

In humans, granuloma formation in response to $M$. tuberculosis infection is essential for control of mycobacterial infections [9]. Paradoxically, granulomatous inflammation is also associated with the typical immunopathology, tissue damage and symptoms seen in TB. It is believed that mycobacterial antigens are continuously released into the infected tissue and could be responsible for the formation and persistence of lesions [10-13]. Antigens secreted into the extracellular environment by $M$. tuberculosis are thought to be immunodominant and to be involved in inducing protective immunity, and in this sense also considered to be the most important antigens during infection $[14,15]$. Little is known about the identity of the M. tuberculosis-antigens that are expressed in vivo in the lesions. $\mathrm{TB}$ is a chronic and multi-organ disease, and the expression profile of antigens in the infected tissues may be different during various phases of infection and in various organs, making it important to study the differential in vivo-expressed antigens in various organs with different disease manifestation.

Previous work has identified and purified major secretory and somatic $M$. tuberculosis antigens to near homogeneity from the culture filtrates and bacterial sonicates from M. tuberculosis cultured in wholly synthetic liquid media [16-18]. Functional rabbit polyclonal antibodies have been generated against these antigens $[17,19,20]$. This collection of antibodies was used to study the in situ M. tuberculosis antigens expression in the infected host tissues. We have previously performed several studies on MPT64 for the development of immunohistochemical method using patient biopsies and aspirate samples to diagnose extrapulmonary TB [21-24]. In this study, we have focused on exploring the expression pattern of several additional major secreted (MPT32, MPT44, MPT46, MPT51, MPT53, MPT59, MPT63, and MPT64) and somatic mycobacterial antigens (Mce1A, Hsp65, and MPT57) and studied the differential expression in the host tissues during advanced rapidly progressing and fatal pulmonary disease with mainly pneumonic infiltrates, and compared this to the presence of the same antigens in TB lymphadenitis cases, which is mainly chronic and self-limiting disease with organised granulomas.

\section{Methods}

\section{Study patients and controls}

Seventeen cases of human tuberculous lymphadenitis and three cases of human pulmonary TB were studied. The patients had either pulmonary or lymph node TB. All the TB lymphadenitis cases had typical histological features of necrotic granulomas consistent with the diagnosis of TB. These were also confirmed as TB cases based on the combined clinical, microbiological, and histological criteria. The pulmonary TB cases were confirmed based on the histology and the detection of tuberculous bacilli from the lesions. Both types of cases were further confirmed to be caused by M. tuberculosis complex organisms by positive nested-PCR based on the amplification of IS6110 as described earlier [22]. Negative controls included 9 foreign-body granulomas of the skin, and 1 each from colon cancer, normal tonsillar tissue, and lung tissue from ischemic heart disease. Based on the clinical information, latent or active TB was excluded from the controls. The controls were further confirmed to lack M.tuberculosis infection by negative nested-PCR of IS6110, negative histology and negative ZN staining. Table 1 shows the baseline features of patients and controls.

\section{Ethics statement}

Ethical clearance was obtained from the regional ethical committee of western Norway. The ethical committee

Table 1 Baseline features of the study patients and controls

\begin{tabular}{|c|c|c|}
\hline & TB cases & Controls \\
\hline \multicolumn{3}{|l|}{ Age group } \\
\hline Children (2-14 years) & 6 & 0 \\
\hline Adults (18-86 years) & 14 & 12 \\
\hline \multicolumn{3}{|l|}{ Gender } \\
\hline Male & 12 & 6 \\
\hline Female & 8 & 6 \\
\hline \multicolumn{3}{|l|}{ Origin of biopsies } \\
\hline $\begin{array}{l}\text { Lung tissues from pulmonary TB with } \\
\text { many AFBs }\end{array}$ & 3 & \\
\hline Cervical lymph nodes & 13 & \\
\hline Axillary lymph nodes & 2 & \\
\hline Mesenteric lymph nodes & 1 & \\
\hline Inguinal lymph nodes & 1 & \\
\hline Foreign-body granulomas of the skin & & 9 \\
\hline Colon cancer & & 1 \\
\hline Normal tonsillar tissue & & 1 \\
\hline $\begin{array}{l}\text { Lung tissues from autopsy of ischemic } \\
\text { heart disease }\end{array}$ & & 1 \\
\hline
\end{tabular}


provided exemption for the written informed consent statement for this study as the biopsies were obtained from a bio-bank consisting of already collected anonymised biomaterial for research purposes.

\section{Histology and immunohistochemistry}

Parallel $5 \mu \mathrm{m}$ thick sections from each specimen were stained with haematoxylin and eosin, and immunostaining using the EnVision + System-HRP kit (DakoCytomation Denmark A/S, Glostrup, Denmark) was done as described previously [22]. Briefly, after deparaffinisation and rehydration, the sections were exposed to microwave-antigenretrieval using citrate buffer pH6.0 at $750 \mathrm{~W}$ for $10 \mathrm{~min}$, and at $350 \mathrm{~W}$ for $15 \mathrm{~min}$. The sections were cooled for $20 \mathrm{~min}$ at room temperature and then incubated with $\mathrm{H}_{2} \mathrm{O}_{2}$ solution for $5 \mathrm{~min}$. Primary antibodies were then applied to the sections for $45 \mathrm{~min}$, and subsequently incubated for 40 min with anti-rabbit immunoglobulin conjugated with dextran polymer and horseradish peroxidase (DakoCytomation Denmark A/S, Glostrup, Denmark). Visualisation was achieved using 3-amino-9-ethylcarbazol (AEC) containing $\mathrm{H}_{2} \mathrm{O}_{2}$ (DakoCytomation Denmark A/S, Glostrup, Denmark). Primary antibodies and dilutions used are shown in Table 2. Two negative controls were used; in the first, primary antibody was substituted with antibody diluent, and in the second, an irrelevant rabbit polyclonal antibody was used. In addition, pre-immune sera for some of the antibodies, and anti-GST anti-sera for anti-Mce1A, were used as controls.

\section{Antibodies}

In-house antibodies were used in the study. These antibodies were raised by immunization of rabbits with antigens purified from $M$. tuberculosis as described earlier $[17,20]$. Briefly, M. tuberculosis H37Rv (ATCC 27294), obtained from the National Institute of Health, Tokyo, Japan, was grown in wholly synthetic Sauton medium. The bacilli were removed by centrifugation and the culture supernatant was concentrated by $80 \%$ ammonium sulphate precipitation. The bacilli were washed three times in phosphate-buffered saline and sonicated in a rosette cooling cell with a model Branson B12 Sonifier. The antigens were purified by combining gel filtration and ion-exchange methods as described previously [17]. Polyclonal rabbit antibodies were obtained by immunising with soluble purified antigen using a standard immunization procedure with Freunds incomplete adjuvant [25]. Table 2 summarises the antibodies used and their specificities.

\section{Evaluation of immunostaining}

Number of stained cells and total nuclei were counted in the lesions using a $\times 40$ ocular fitted with a $10 \times 10 \mathrm{~mm}$ grid. One section from each individual case and three areas per section were counted. It was difficult to have exact counts as many positive cells were clustered together. Due to error in the counting of positive cells and large intra-observer variability, it was chosen to present data as semi-quantitative analysis instead of exact numbers/percentages, where " +++++ " was used to describe

Table 2 Antibodies used in the immunohistochemical staining

\begin{tabular}{|c|c|c|c|}
\hline Antibody & Target antigen & Protein function & Dilution \\
\hline \multicolumn{4}{|l|}{$\begin{array}{l}\text { Secreted } \\
\text { antigens }\end{array}$} \\
\hline Anti-MPT32 & MPT32 (Apa, Rv1860) & Unknown (Could mediate bacterial attachment to host cells) & $1: 500$ \\
\hline Anti-MPT44 & $\begin{array}{l}\text { MPT44 (Ag85A, fbpA, } \\
\text { Rv3804c) }\end{array}$ & $\begin{array}{l}\text { Involved in cell wall mycoloylation. Responsible for the high affinity of mycobacteria to } \\
\text { fibronectin. Possesses a mycolyltransferase activity required for the biogenesis of trehalose } \\
\text { dimycolate (cord factor), a dominant structure necessary for maintaining cell wall integrity. }\end{array}$ & 1:100 \\
\hline Anti-MPT46 & $\begin{array}{l}\text { MPT46 (Thioredoxin } \\
\text { TrxC, Rv3914) }\end{array}$ & $\begin{array}{l}\text { Participates in various redox reactions through the reversible oxidation of its active } \\
\text { center dithiol, to a disulfide, \& catalyzes dithiol-disulfide exchange reactions. }\end{array}$ & 1:500 \\
\hline Anti-MPT51 & $\begin{array}{l}\text { MPT51 (Ag85D, fbpD, } \\
\text { Rv3803c)/TB22.2 }\end{array}$ & Same as MPT44 & 1:500 \\
\hline Anti-MPT53 & MPT53 (DsbE, Rv2878c) & Unknown & 1:500 \\
\hline Anti-MPT59 & $\begin{array}{l}\text { MPT59 (Ag85B, fbpB, } \\
\text { Rv1886c) }\end{array}$ & Same as MPT44 & 1:100 \\
\hline Anti-MPT63 & MPT63 (Rv1926c) & Unknown & $1: 500$ \\
\hline Anti-MPT64 & MPT64 (Rv1980c) & Unknown (Suggested to inhibit apoptosis of infected cells) & $1: 250$ \\
\hline \multicolumn{4}{|l|}{$\begin{array}{l}\text { Somatic } \\
\text { antigens }\end{array}$} \\
\hline Anti-Mce1A & Mce1A (Rv0169) & Thought to be involved in cell invasion (entry and survival) & $1: 2000$ \\
\hline Anti-Hsp65 & $\begin{array}{l}\text { Hsp65 (Ag82, GroEL2, } \\
\text { Rv0440) }\end{array}$ & $\begin{array}{l}\text { Prevents misfolding and promotes the refolding and proper assembly of unfolded } \\
\text { polypeptides generated under stress conditions. }\end{array}$ & 1:500 \\
\hline Anti-MPT57 & MPT57 (GroES, Rv3418c) & Binds to Cpn60 in the presence of Mg-ATP and suppresses the ATPase activity of the latter. & $1: 4000$ \\
\hline
\end{tabular}


lesions where $\geq 80 \%$ granuloma cells were positive, " ++++ " if about $50 \%$ granuloma cells were positive, "+++" if 10$25 \%$ granuloma cells were positive, "++" if 2-9\% granuloma cells were positive, "+" if $<2 \%$ granuloma cells were positive, and "-" if no positive cells were detected. A qualitative description of the staining pattern was also done. A cut-off of positive cells to define a positive case was not used since extrapulmonary TB is usually paucibacillary. The staining in negative controls was instead discriminated as nonspecific based on the quality and location of staining.

\section{Nested polymerase chain reaction for IS6110}

Five to six, $8 \mu \mathrm{m}$ sections from each paraffin embedded tissue blocks were collected in sample preparation tubes for nested PCR. Carry-over tissue contamination was prevented by cleaning the blade with $96 \%$ ethanol after sectioning each sample; negative controls were sectioned first, followed by test blocks and positive control blocks.

DNA extraction and nested PCR on paraffin sections were performed as described previously [22]. Briefly, following proteinase $\mathrm{K}$ digestion, bacterial genomic DNA was eluted in water using a MagAttract DNA mini M48 Kit (Qiagen,West Sussex, UK) on Biorobot M48 (Qiagen). A 123- base pair fragment from IS6110 was amplified using the following primers $5^{\prime}$ CCTGCGAGCGTAGGC GTCGG 3' and 5' CTCGTCCAGCGCCGCTTCGG 3'. The product was subjected to a second round of PCR amplification using the primers 5' TTCGGACCACCAGC
ACCTAA 3' and 5' TCGGTGACAAAGGCCACGTA 3' to amplify a 92-base pair fragment. The PCR reaction mixture consisted of $5 \mu \mathrm{l}$ eluted DNA, $25 \mu \mathrm{l}$ of HotStarTaq master mix (Qiagen), $0.25 \mu$ l of each $100 \mu \mathrm{M}$ primer stock solution, distilled water to make a final volume of $50 \mu \mathrm{l}$. For nested PCR, $1 \mu \mathrm{l}$ of the first PCR product was used as template. The reaction cycle for the first PCR was- $94^{\circ} \mathrm{C}$ for 1 minute, $68^{\circ} \mathrm{C}$ for 1 minute, $72^{\circ} \mathrm{C}$ for 20 seconds for 45 cycles and for the nested PCR $-94^{\circ} \mathrm{C}$ for 1 minute, $58^{\circ} \mathrm{C}$ for 1 minute, $72^{\circ} \mathrm{C}$ for 20 seconds for 35 cycles. Both PCR's had an initial heat activation step of $95^{\circ} \mathrm{C}$ for 15 minutes and a final extension of $72^{\circ} \mathrm{C}$ for 10 minutes. The amplified product was analyzed in a $3 \%$ agarose gel stained with ethidium bromide. Mycobacterial DNA, and positive PCR product were included as positive controls and an extraction control (with all the steps but without any tissue), a reaction tube with substitution of distilled water for the test template and a sample which previously yielded negative result on PCR were included as negative control in each PCR run.

\section{Results}

Morphology, acid-fast staining and PCR

All the lymph node biopsies had necrotic granulomatous inflammation consistent with TB. There were focal aggregates of epithelioid macrophages and multi-nucleated giant cells with a central necrotic area. The lung tissues showed widespread pneumonic infiltrates with chronic

Table 3 Immunohistochemical analysis of in vivo expression of various mycobacterial antigens in the formalin fixed tissues obtained from Mycobacterium tuberculosis-infected lungs and lymph nodes, and non-tuberculous controls

\begin{tabular}{llll}
\hline & & No. with positive results/total number (\%)*** & Non-TB controls \\
\cline { 2 - 4 } & Multibacillary lung tissues** & Tuberculous lymphadenitis & $0 / 12(0)$ \\
ZN staining & $3 / 3(100)$ & $0 / 17(0)$ & $0 / 12(0)$ \\
Secreted antigens & $3 / 3(100)$ & $17 / 17(100)$ & $0 / 12(0)$ \\
MPT32 & & & $6 / 12(50)$ \\
MPT44 & $3 / 3(100)$ & $3 / 17(18)$ & $1 / 12(8)$ \\
MPT46 & $1 / 3(33)$ & $4 / 14(28)$ & $0 / 12(0)$ \\
MPT51 & $3 / 3(100)$ & $0 / 17(0)$ & $1 / 12(8)$ \\
MPT53 & $3 / 3(100)$ & $0 / 17(0)$ & $3 / 12(25)$ \\
MPT59 & $3 / 3(100)$ & $3 / 17(18)$ & $2 / 12(17)$ \\
MPT63 & $3 / 3(100)$ & $6 / 17(35)$ & $2 / 12(17)$ \\
MPT64 & $3 / 3(100)$ & $2 / 17(12)$ & $17 / 17(100)$ \\
Somatic antigens & $3 / 3(100)$ & & $8 / 12(67)$ \\
Mce1A & & $17 / 17(100)$ & $12 / 12(100)$ \\
Hsp65 & $3 / 3(100)$ & $17 / 17(100)$ & $12 / 12(100)$ \\
MPT57 & $3 / 3(100)$ & $16 / 16(100)$ & \\
\hline
\end{tabular}

*PCR, N-PCR for amplification of IS6110.

**Vast number of bacilli were detectable by ZN staining.

${ }^{* * *}$ A case/control was labelled as positive if any positive signal was recorded in a lesion irrespective of the quality of stain. 
inflammatory cells, but lacking the necrotic granuloma morphology seen in lymphadenitis lesions. Plenty of bacilli were detectable with acid fast staining in the lung biopsies while no bacilli were detected by this method in the lymph node biopsies. All the cases were shown to be positive by nested PCR for IS6110, which is specific for M. tuberculosis complex organisms.

\section{Secreted mycobacterial antigens in lung tissues}

The major secreted antigens; MPT32, MPT44 (Ag85A), MPT46, MPT51 (Ag85D), MPT53, MPT59 (Ag85B), MPT63, and MPT64 were detected by strong staining in all pulmonary infiltrates (Tables 3 and 4). The presence of antigens was primarily seen as intracellular granular staining in the cytoplasm of inflammatory cells (Figure 1). The stained cells were uniformly distributed in the lesions. MPT44 was found in a lower number of cells as compared to other antigens. Table 3 shows the number of positive cases for each antigen.

\section{Secreted mycobacterial antigens in lymph nodes}

All secreted antigens described above were detected at a low level, or were absent, in the tuberculous lymphadenitis cases. The exception was MPT64, which were observed in all of the lymphadenitis cases, with variable staining intensities (Tables 3 and 4, Figure 2). Components of the antigen 85 complex: MPT44 (Ag85A), MPT51 (Ag85D), MPT59 (Ag85B), which constitute a large proportion of in vitro-secreted antigens, were identified in only $8 / 17$ of cases. Among the positive cases, staining was found in less than $2 \%$ of granuloma cells, or only in the necrotic centres - typically in one or two out of 5-7 granulomas in a lymph node section (Table 4, Figure 2). The staining pattern was granular with strong intensity and seen as discrete localised stained areas (Figure 2). Despite the homology between various components of the antigen 85 complex, their staining pattern differed in this tissue. MPT44 $(\operatorname{Ag} 85 \mathrm{~A})$ was seen in 4 cases; it was detected in less than $2 \%$ of granuloma cells in two of the cases, and only in necrotic centres in the other 2 cases. MPT59 (Ag85B) was detected in the necrotic centres in 4 cases. There was no agreement between the cases where these antigens were found. MPT51 (Ag85D) was not detected in any of the investigated sections. MPT53 was only found in necrotic centres of granulomas in three cases, and the

Table 4 Semiquantitative comparative analysis of the various mycobacterial antigens in the individual cases of pulmonary and lymph node tuberculous detected by immunohistochemistry done on formalin fixed paraffin embedded tissues

\begin{tabular}{|c|c|c|c|c|c|c|c|c|c|c|c|}
\hline & \multicolumn{8}{|c|}{ Secreted antigens } & \multicolumn{3}{|c|}{ Somatic antigens } \\
\hline & МРT32 & MPT44 & MPT46 & MPT51 & MPT53 & MPT59 & MPT63 & MPT64 & Mce1A & Hsp65 & MPT57 \\
\hline Lung 1 & +++++ & - & +++++ & +++++ & +++++ & ++++ & +++++ & +++++ & +++ & +++++ & ++++ \\
\hline Lung 2 & +++++ & - & +++++ & +++++ & +++++ & ++++ & +++++ & +++++ & +++ & ++ & ++ \\
\hline Lung 3 & +++ & +++ & +++++ & ++++ & ++++ & ++++ & ++++ & ++++ & ++ & ++ & +++ \\
\hline LN 1 & - & - & - & - & - & SNC & - & + & + & +++ & +++ \\
\hline LN 2 & - & SNC & - & - & - & - & - & + & +++ &,$+++ \mathrm{SNC}$ & +++ \\
\hline LN 3 & - & - & - & - & - & - & - & + & ++ & ++++ & ++++ \\
\hline LN 4 & - & - & - & - & - & - & - & + & + & ++++ & + \\
\hline LN 5 & - & - & - & - & - & - & - & + &,+++ SNC & +++++ & +++++ \\
\hline LN 6 & + & - & - & - & - & SNC & - & ++ & ++ & ++++ & ++++ \\
\hline LN 7 & - & - & - & - & - & SNC & - & ++ & ++ & +++ & + \\
\hline LN 8 & - & ND & - & - & SNC & SNC & - & ++ & + & +++ & +++ \\
\hline LN 9 & - & ND & - & - & - & - & - & ++ & ++ & +++ & +++ \\
\hline LN 10 & - & + & - & - & - & - & - & ++ & + & +++ & ++++ \\
\hline LN 11 & - & ND & - & - & - & + & - & ++ & + & +++ & + \\
\hline LN 12 & - & - & - & - & SNC & - & + & ++ & +++ & +++ & +++ \\
\hline LN 13 & + & - & - & - & - & + & - & +++ & + & +++++ & + \\
\hline LN 14 & - & - & - & - & - & - & - & +++ & + & +++ & +++ \\
\hline LN 15 & - & + & - & - & SNC & - & - & +++ & +++ & ++++ & ++++ \\
\hline LN 16 & + & SNC & - & - & - & - & + & +++ & ++ & ++ & ND \\
\hline LN 17 & - & - & - & - & - & - & - & ++ & + & ++ & ++++ \\
\hline
\end{tabular}

LN: Lymph node, SNC; staining in the necrotic centres, ND; not determined.

$+++++\sim 80 \%$ positive granuloma cells, $++++\sim 50 \%$ positive granuloma cells, $+++10-25 \%$ positive granuloma cells, $++2-9 \%$ positive granuloma cells, $+<2 \%$ positive granuloma cells, - no staining. 

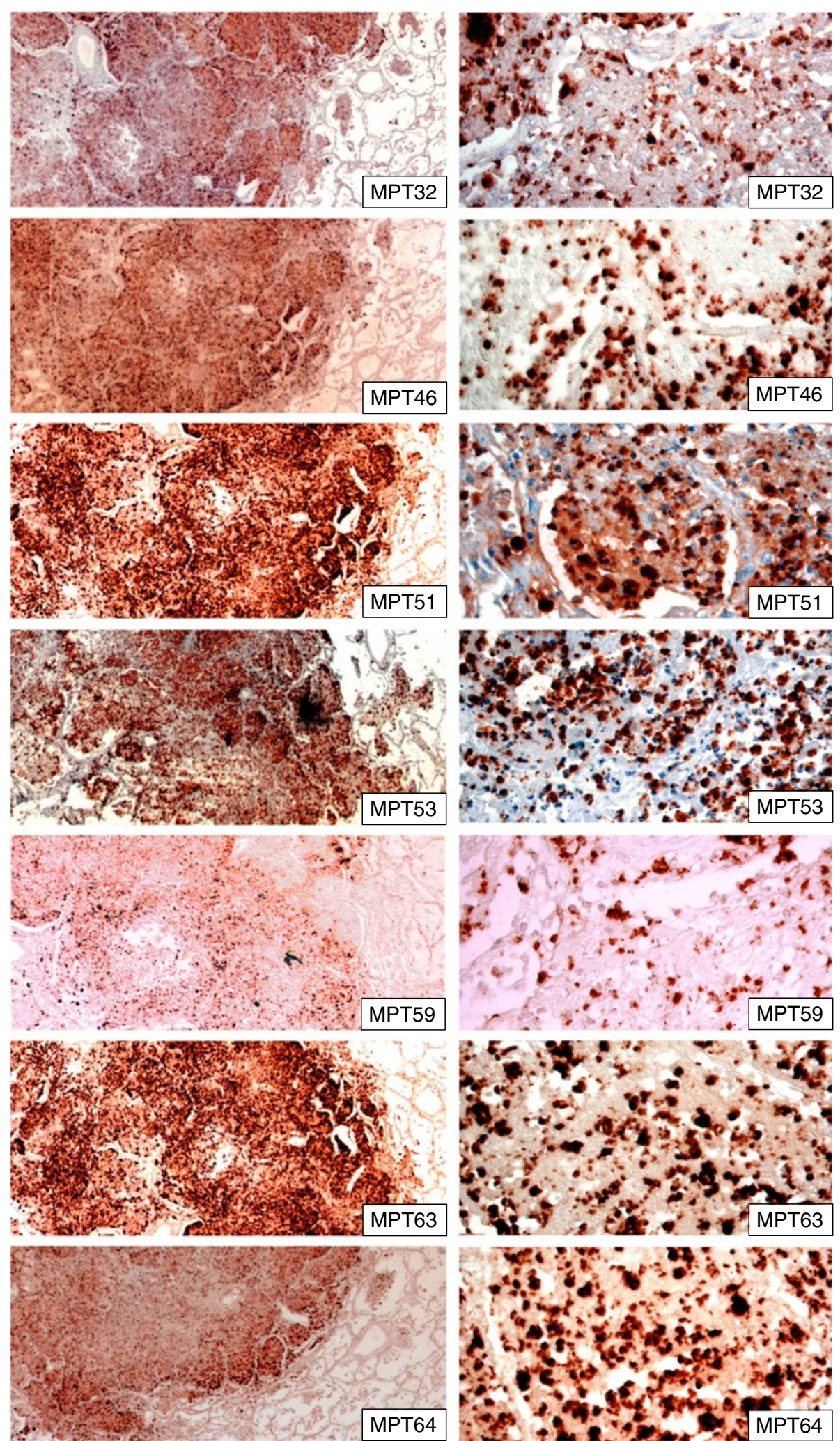

Figure 1 (See legend on next page.) 
(See figure on previous page.)

Figure 1 Human pulmonary tissues infected with $M$. tuberculosis showing the staining pattern of secreted mycobacterial antigens in the lesions as detected by immunohistochemical staining. The right column shows a higher magnification of corresponding pictures, and illustrates intracellular location of all antigens and the granular staining pattern. All antigens are expressed in abundance in the lesions. The staining pattern varies and MPT59 (Ag85B) was detected less frequently than other antigens. The staining is detected mainly in the inflammatory lesions, but a few macrophages in close proximity of the lesions were also stained by these antibodies.

staining pattern was similar to that of MPT59 (Ag85B) (Figure 2). MPT63 and MPT32 were identified in 2 and 3 cases respectively in less than $2 \%$ of granuloma cells, while MPT46 was not found in any of the lymphadenitis cases. (Tables 3 and 4).

\section{Somatic mycobacterial antigens in lung tissues}

There was a difference in the staining patterns obtained using antibodies against somatic and secreted antigens. In the pulmonary lesions, a lower ratio of cells was stained for somatic antigens as compared to the secreted antigens
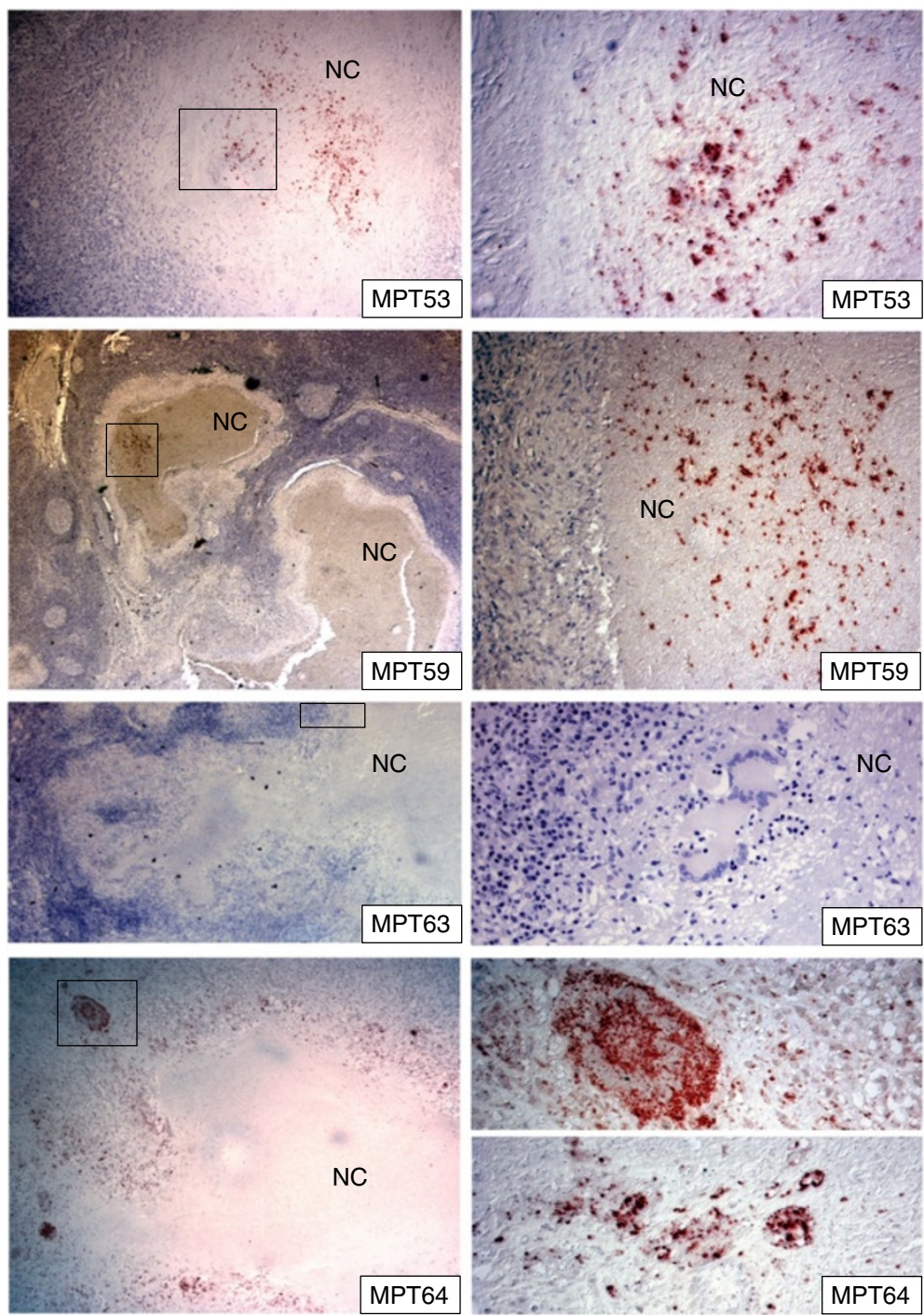

Figure 2 Human lymph nodes tissue infected with $M$. tuberculosis showing the staining pattern of mycobacterial secreted antigens in the necrotic granulomas as detected by immunohistochemical staining. Right column shows higher magnification of the marked areas in the corresponding pictures. There is a differential staining of various secreted antigens. MPT64 is detected in epithelioid cells and multinucleated giant cells, while the necrotic centres do not contain this antigen. MPT59 (Ag85B) and MPT63 were detected in few cases and were located only in the necrotic centres in these cases. Granuloma cells were not stained by these antibodies. The majority of the lymphadenitis granulomas were not stained for secreted antigens. NC = necrotic centres. 


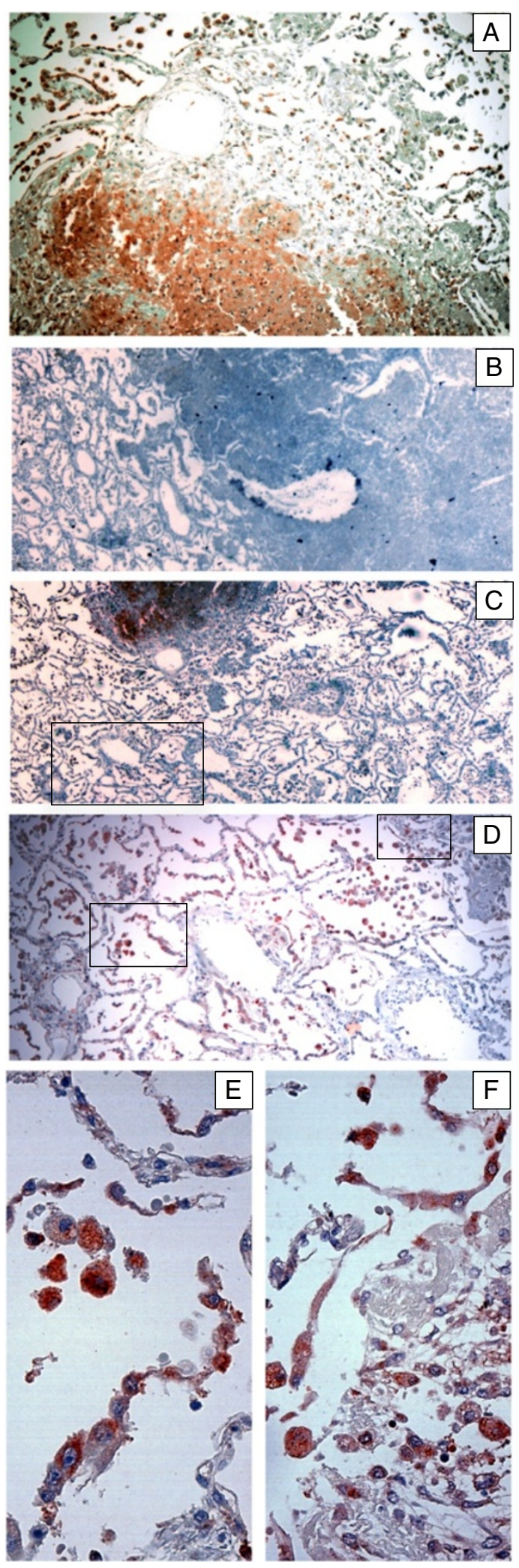

Figure 3 Staining pattern of mycobacterial Mce1A antigen in human pulmonary tissues infected with $M$. tuberculosis as detected by immunohistochemical staining. Panels $\mathbf{A}-\mathbf{C}$ shows three different infiltrates from the same lung section, the area marked in $\mathbf{C}$ is shown in higher magnification in $\mathbf{D}$ and the two demarcated areas in panel $\mathbf{D}$ are magnified in panels $\mathbf{E}-\mathbf{F}$. The expression of Mce $1 \mathrm{~A}$ was relatively lower as compared to the other secreted antigens. One of the infiltrates did not contain this antigen, while the expression of other secreted antigens was abundant in this infiltrate (as shown in Figure 1). The other two infiltrates expressed this antigen at a relatively lower level as compared to the other secreted antigens. The lung parenchyma in vicinity of the infiltrates expressed this antigen more abundantly as compared to the other secreted antigens. Alveolar macrophages, activated macrophages, and alveolar lining cells expressed the antigen.

and the intensity of staining was weaker as compared to the staining of the secreted antigens. In some areas, staining of anti-Mce1A would co-localise with that of the secreted antigens, while in other regions the anti-Mce1A staining was not detected despite the presence of a strong staining for all secreted antigens. Furthermore, cells stained by anti-Mce1A were observed in areas of the lungs without pneumonic infiltrates while secreted antigens were not stained in these areas (Figure 3).

\section{Somatic mycobacterial antigens in lymph node tissues}

In the tuberculous lymphadenitis granulomas, somatic antigens were detected in all the cases. Antigens were primarily found in the granuloma-, epithelioid- and multinucleated giant cells, and very little in necrotic centres (Figure 4). The staining was granular and found within the host cell cytoplasm with strong intensity. The number of stained cells varied among the cases as shown in Table 4. Hsp65 was detectable in a higher number of cells as compared to other antigens (Tables 3 and 4, Figure 4). The pattern of staining for MPT57 (GroES) was less granular and more diffuse than what was observed for the other two somatic antigens. In the granulomas, multinucleated giant cells were not stained as strongly by anti-MPT57 as they were by anti-Mce1A or anti-Hsp65 (Figure 4).

Among the negative controls, several cases also showed positive signals and they were recorded as positive, but here the intensity of staining was weaker and the staining pattern was diffuse rather than granular. Furthermore, the staining found in non-inflammatory cells was located in the nucleus and not in the cytoplasm, and it was primarily observed in the periphery of the section. Based on these differences in the quality and location of staining pattern the positive antigen staining in the negative controls was concluded as non-specific and it was possible to discriminate that by an expert pathologist. However this information of non-specific staining as positive signals is included in the results in order to highlight that which of the 


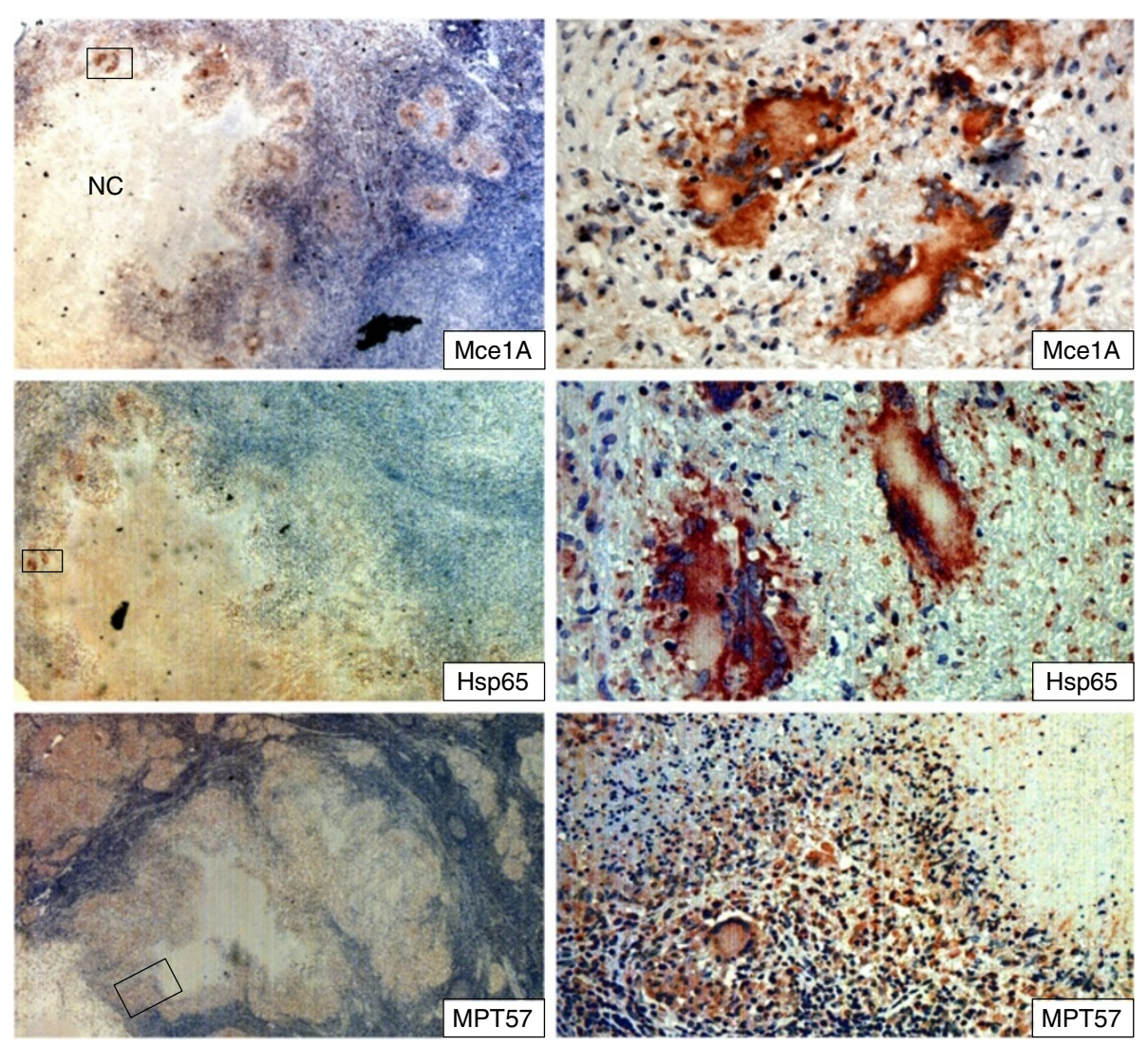

Figure 4 Staining pattern of mycobacterial cell membrane (Mce1A) and somatic (MPT57 and Hsp65) antigens in lymph nodes tissues infected with $\boldsymbol{M}$. tuberculosis, as detected by immunohistochemical staining. The areas marked in the left column panels are shown in higher magnification in the right column panels. Mce1A and Hsp65 was stained strongly in epithelioid cells and multinucleated giant cells, while the staining intensity of MPT 57 was weaker in these cells. Mce1A was not detected in the necrotic centres. NC = necrotic centres.

antigens/antibodies is better suited for use as a diagnostic test, where the interpretation should be more robust.

\section{Discussion}

In this study, we analysed the expression of mycobacterial antigens in infected tissues.

One interesting observation was the differential staining pattern of secreted antigens in the lungs and lymph node lesions. While these antigens were found in high amounts in the pulmonary lesions, their level of staining was very low, or absent, in the lymph node granulomas. This contrasted the findings for somatic antigens, which were found with the same ratio in both types of lesions. The pulmonary lesions exhibited pneumonic infiltrates without proper necrotic granuloma formation, while the lymph node lesions had organised necrotic granulomas. Granuloma formation is known to be a correlate of an effective immune response resulting in control of bacillary multiplication and containment of infection. Relative absence of secreted mycobacterial antigens in the well-formed granulomas indicate that immune response is effective to bring bacillary multiplication under control, while the presence of somatic antigens imply their involvement in the persistence of infection.

We could not detect bacilli by acid-fast staining in the lymph node tissues, which might indicate that the number of intact bacilli is below a detectable level. However, it has been shown that mycobacteria tend to lose of their acid-fastness as they adapt to the stressful intracellular milieu within a granuloma [26-28]. This can provide an alternative explanation to why no bacilli could be detected in the lymph nodes using this technique. The mycobacterial response to stressful conditions is multifactorial. Other observed change is the down-regulation of a number of secreted antigens that are considered to be highly expressed during normal culture conditions, including Ag85A-C, MPT63 and MPT64 [29-31]. As the bacteria adapt to persistence and long-term survival within cells of the granuloma, many of the antigens required during, and immediately after, cell invasion, are no longer important for bacterial survival. This could explain why most of the investigated secreted antigens are nearly absent in the lymph nodes, while the somatic antigens were equally detected in both lung and lymph node tissues. Serodiagnostic 
studies have evaluated specificities of antibodies from sera of TB patients against different mycobacterial antigens including secreted-, membrane-, cell wall associated-, and cytosolic antigens. Although secreted antigens have been most commonly studied, it is the surface exposed components that appear to be most frequently recognised by patient sera [32,33]. A study that described a lack of host immune response towards the secreted mycobacterial antigen MPT53 in humans and guinea pigs [34], lends support to the theory of a reduced presence of secreted antigens in vivo during stable disease. The question remains why the major secreted antigens in some cases were only detected in the necrotic centres and not in the granuloma cells. One possible explanation is that the discrete spots of staining in the necrotic centres represent cells that harbour multiplying bacilli that produce secreted antigens, but that these cells are gradually removed by cell lysis and necrosis.

There was a notable exception to the almost complete absence of staining of secreted antigens in the lymphadenitis tissues; the antigen MPT64 was identified in all granulomas. The explanation for this observation could be that MPT64 is not cleared away from the tissues and therefore accumulates in the granulomas over time. Structural analyses of this protein, suggest that it harbours a $\beta$-grasp motif that can form strong protein-protein interactions $[35,36]$. We have previously studied the staining pattern of MPT64 in tuberculous pleural biopsies in HIV co-infected TB patients, where only about half of the biopsies had organised granulomatous inflammation. MPT64 antigen was more frequently detected in the granulomas than in the non-granulomatous inflammation (93\% vs. $64 \%$ ), while mycobacterial DNA was detected more frequently in the non-granulomatous inflammation as compared to granulomas [21]. This antigen therefore seem to preferentially accumulate within granuloma structures, even though the cells of the granuloma are efficiently restricting bacterial growth. The persistence of MPT64 in the tissues suggests a role in bacterial pathogenesis.

In pulmonary lesions without proper granuloma formation, the ratio of cells that stained positive for the somatic antigen Mce1A was lower, and the staining intensity was weaker, compared to the staining pattern of secreted antigens. Furthermore, the staining pattern of Mce1A and secreted antigens did not always overlap; certain regions were not stained by anti-Mce1A, despite being highly stained by antibodies against secreted antigens. In lymph nodes with organised granulomas, Mce1A was detected in all cases, located mainly intracellularly in the granuloma cells rather than necrotic centres as seen with some secreted antigens. The products of the $m c e 1$ operon have been shown to be important for granuloma formation [37-39]. In this study a differential staining pattern of Mce1A in lungs with excessive pathology, but without the typical organised granulomas as compared to lymph node tissue, lend support to the theory of an involvement of this antigen in the formation of organised and protective granulomas.

\section{Conclusions}

This study shows that major secreted mycobacterial antigens were found in high amounts in the pulmonary lesions that exhibited pneumonic infiltrates without proper necrotic granuloma formation, while their level of staining was very low, or absent, in the lymph node TB lesions with organised necrotic granulomas and very low bacillary load. One exception was MPT64, which was detected in both pulmonary and lymph node lesions. This indicates a preferential accumulation of this antigen within granuloma structures even if the cells of the granuloma can efficiently restrict bacterial growth, and suggests a role in the persistence of chronic infection. All three somatic antigens were detectable in both pulmonary and lymphadenitis lesions, and their expression pattern was different from that of the secreted antigens. The findings in this study have implications for the development of new diagnostic tools.

\section{Competing interests}

The authors declare that they have no competing interests.

\section{Authors' contributions}

TM conceived and designed the study, carried out the experiments, analysed the data and drafted the manuscript. NAL participated in the experiments, and drafting of the manuscript. LS participated in the analysis of the immunohistochemistry, and drafting of the manuscript. HGW participated in the antibody preparation, and drafting of the manuscript. All authors read and approved the final manuscript.

\section{Acknowledgments}

This work was supported by funds from the Norwegian Research Council. We thank Kalaiarasy Kugarajh for technical assistance.

\section{Author details}

${ }^{1}$ Centre for International Health, Department of Global Public Health and Primary Care, University of Bergen, Bergen, Norway. ${ }^{2}$ Department of Thoracic Medicine, Haukeland University Hospital, Bergen, Norway. ${ }^{3}$ Department of Clinical Science, University of Bergen, Bergen, Norway. ${ }^{4}$ Section for Pathology, Department of Clinical Medicine, University of Bergen, Bergen, Norway. ${ }^{5}$ Department of Pathology, Haukeland University Hospital, Bergen, Norway. ${ }^{6}$ Department of Microbiology, Haukeland University Hospital, Bergen, Norway.

Received: 8 May 2014 Accepted: 22 September 2014

Published: 3 October 2014

\section{References}

1. World Health Organization: Global tuberculosis report. Geneva, Switzerland: WHO Press; 2012.

2. Small PM, Schecter GF, Goodman PC, Sande MA, Chaisson RE, Hopewell PC: Treatment of tuberculosis in patients with advanced human immunodeficiency virus infection. N Engl J Med 1991, 324:289-294.

3. Clark RA, Blakley SL, Greer D, Smith MH, Brandon W, Wisniewski TL: Hematogenous dissemination of Mycobacterium tuberculosis in patients with AIDS. Rev Infect Dis 1991, 13:1089-1092.

4. Seibert AF, Haynes J Jr, Middleton R, Bass JB Jr: Tuberculous pleural effusion. Twenty-year experience. Chest 1991, 99:883-886.

5. Marais BJ, Pai M: Recent advances in the diagnosis of childhood tuberculosis. Arch Dis Child 2007, 92:446-452. 
6. Peto HM, Pratt RH, Harrington TA, LoBue PA, Armstrong LR: Epidemiology of extrapulmonary tuberculosis in the United States, 1993-2006. Clin Infect Dis 2009, 49:1350-1357.

7. Wilsher ML, Hagan C, Prestidge R, Wells AU, Murison G: Human in vitro immune responses to Mycobacterium tuberculosis. Tuber Lung Dis 1999, 79:371-377.

8. Hussain R, Toossi Z, Hasan R, Jamil B, Dawood G, Ellner JJ: Immune response profile in patients with active tuberculosis in a BCG vaccinated area. Southeast Asian J Trop Med Public Health 1997, 28:764-773.

9. Emile JF, Patey N, Altare F, Lamhamedi S, Jouanguy E, Boman F, Quillard J, Lecomte-Houcke M, Verola O, Mousnier JF, Dijoud F, Blanche S, Fischer A, Brousse N, Casanova JL: Correlation of granuloma structure with clinical outcome defines two types of idiopathic disseminated BCG infection. J Pathol 1997, 181:25-30.

10. Barbolini G, Bisetti A, Colizzi V, Damiani G, Migaldi M, Vismara D: Immunohistologic analysis of mycobacterial antigens by monoclonal-antibodies in tuberculosis and mycobacteriosis. Hum Pathol 1989, 20:1078-1083.

11. Fenhalls G, Stevens L, Moses L, Bezuidenhout J, Betts JC, Helden Pv P, Lukey PT, Duncan K: In situ detection of Mycobacterium tuberculosis transcripts in human lung granulomas reveals differential gene expression in necrotic lesions. Infect Immun 2002, 70:6330-6338.

12. Fenhalls G, Stevens-Muller L, Warren R, Carroll N, Bezuidenhout J, van Helden P, Bardin P: Localisation of mycobacterial DNA and mRNA in human tuberculous granulomas. J Microbiol Methods 2002, 51:197-208.

13. Goel MM, Budhwar P: Immunohistochemical localization of mycobacterium tuberculosis complex antigen with antibody to $38 \mathrm{kDa}$ antigen versus Ziehl Neelsen staining in tissue granulomas of extrapulmonary tuberculosis. Indian J Tuberc 2007, 54:24-29.

14. Andersen P: Host responses and antigens involved in protective immunity to Mycobacterium tuberculosis. Scand J Immunol 1997, 45:115-131.

15. Wiker HG, Harboe M, Lea TE: Purification and characterization of two protein antigens from the heterogeneous BCG85 complex in Mycobacterium bovis BCG. Int Arch Allergy Appl Immunol 1986, 81:298-306.

16. Målen H, Berven FS, Fladmark KE, Wiker HG: Comprehensive analysis of exported proteins from Mycobacterium tuberculosis. Proteomics 2007, 10:1702-1718

17. Nagai S, Wiker HG, Harboe M, Kinomoto M: Isolation and partial characterization of major protein antigens in the culture fluid of Mycobacterium tuberculosis. Infect Immun 1991, 59:372-382.

18. de Souza GA, Malen H, Softeland T, Saelensminde G, Prasad S, Jonassen I, Wiker HG: High accuracy mass spectrometry analysis as a tool to verify and improve gene annotation using Mycobacterium tuberculosis as an example. BMC Genomics 2008, 9:316.

19. Harboe M, Malin AS, Dockrell HS, Wiker HG, Ulvund G, Holm A, Jørgensen MC, Andersen P: B-cell epitopes and quantification of the ESAT-6 protein of Mycobacterium tuberculosis. Infect Immun 1998, 66:717-723.

20. Wiker HG, Harboe M, Nagai S: A localization index for distinction between extracellular and intracellular antigens of $M$ tuberculosis. J Gen Microbiol 1991, 137:875-884.

21. Baba K, Dyrhol-Riise AM, Sviland L, Langeland N, Hoosen AA, Wiker HG, Mustafa T: Rapid and specific diagnosis of tuberculous pleuritis with immunohistochemistry by detecting Mycobacterium tuberculosis complex specific antigen MPT64 in patients from a HIV endemic area. Appl Immunohistochem Mol Morphol 2008, 16:554-561.

22. Mustafa T, Wiker HG, Mfinanga SG, Morkve O, Sviland L: Immunohistochemistry using a Mycobacterium tuberculosis complex specific antibody for improved diagnosis of tuberculous lymphadenitis. Mod Pathol 2006, 19:1606-1614.

23. Purohit MR, Mustafa T, Wiker HG, Morkve O, Sviland L: Immunohistochemical diagnosis of abdominal and lymph node tuberculosis by detecting Mycobacterium tuberculosis complex specific antigen MPT64. Diagn Pathol 2007, 2:36.

24. Purohit MR, Mustafa T, Wiker HG, Sviland L: Rapid diagnosis of tuberculosis in aspirate, effusions, and cerebrospinal fluid by immunocytochemical detection of Mycobacterium tuberculosis complex specific antigen MPT64. Diagn Cytopathol 2012, 40:782-791.

25. Harboe $M$, Closs $O$, Deverill J: Production of monospecific antisera against antigenic components of M bovis (BCG). Scand J Immunol 1976, 5:861866.
26. Nyka W: Studies on the effect of starvation on mycobacteria. Infect Immun 1974, 9:843-850.

27. Deb C, Lee CM, Dubey VS, Daniel J, Abomoelak B, Sirakova TD, Pawar S, Rogers L, Kolattukudy PE: A novel in vitro multiple-stress dormancy model for Mycobacterium tuberculosis generates a lipid-loaded, drug-tolerant, dormant pathogen. PLoS One 2009, 4:e6077.

28. Velayati AA, Farnia P, Masjedi MR, Zhavnerko GK, Merza MA, Ghanavi J, Tabarsi P, Farnia P, Poleschuyk NN, Ignatyev G: Sequential adaptation in latent tuberculosis bacilli: observation by atomic force microscopy (AFM). Int J Clin Exp Med 2011, 4:193-199.

29. Schnappinger D, Ehrt S, Voskuil MI, Liu Y, Mangan JA, Monahan IM, Dolganov G, Efron B, Butcher PD, Nathan C, Schoolnik GK: Transcriptional adaptation of mycobacterium tuberculosis within macrophages: insights into the phagosomal environment. J Exp Med 2003, 198:693-704.

30. Rohde KH, Abramovitch RB, Russell DG: Mycobacterium tuberculosis invasion of macrophages: linking bacterial gene expression to environmental cues. Cell Host Microbe 2007, 2:352-364.

31. Aagaard C, Hoang T, Dietrich J, Cardona PJ, Izzo A, Dolganov G, Schoolnik GK, Cassidy JP, Billeskov R, Andersen P: A multistage tuberculosis vaccine that confers efficient protection before and after exposure. Nat Med 2011, 17:189-194.

32. Alifano $M$, De Pascalis $R$, Sofia M, Faraone $S$, Del Pezzo M, Covelli I: Detection of $\lg G$ and IgA against the mycobacterial antigen A60 in patients with extrapulmonary tuberculosis. Thorax 1998, 53:377-380.

33. Abebe F, Holm-Hansen C, Wiker HG, Bjune G: Progress in serodiagnosis of Mycobacterium tuberculosis infection. Scand J Immunol 2007, 66:176-191.

34. Johnson S, Brusasca P, Lyashchenko K, Spencer JS, Wiker HG, Bifani P, Shashkina E, Kreiswirth B, Harboe M, Schluger N, Gomez M, Gennaro ML: Characterization of the secreted MPT53 antigen of Mycobacterium tuberculosis. Infect Immun 2001, 69:5936-5939.

35. Wang Z, Potter BM, Gray AM, Sacksteder KA, Geisbrecht BV, Laity JH: The solution structure of antigen MPT64 from Mycobacterium tuberculosis defines a new family of beta-grasp proteins. J Mol Biol 2007, 366:375-381.

36. Chu TP, Yuann JM: Expression, purification, and characterization of protective MPT64 antigen protein and identification of its multimers isolated from nontoxic Mycobacterium tuberculosis H37Ra. Biotechnol Appl Biochem 2011, 58:185-189.

37. Lima P, Sidders B, Morici L, Reader R, Senaratne R, Casali N, Riley LW: Enhanced mortality despite control of lung infection in mice aerogenically infected with a Mycobacterium tuberculosis mce1 operon mutant. Microbes Infect 2007, 9:1285-1290.

38. Shimono N, Morici L, Casali N, Cantrell S, Sidders B, Ehrt S, Riley LW: Hypervirulent mutant of Mycobacterium tuberculosis resulting from disruption of the mce1 operon. Proc Natl Acad Sci U S A 2003, 100:15918-15923.

39. Uchida Y, Casali N, White A, Morici L, Kendall LV, Riley LW: Accelerated immunopathological response of mice infected with Mycobacterium tuberculosis disrupted in the mce1 operon negative transcriptional regulator. Cell Microbiol 2007, 9:1275-1283.

doi:10.1186/1471-2334-14-535

Cite this article as: Mustafa et al:: Differential in vivo expression of mycobacterial antigens in Mycobacterium tuberculosis infected lungs and lymph node tissues. BMC Infectious Diseases 2014 14:535.

\section{Submit your next manuscript to BioMed Central and take full advantage of:}

- Convenient online submission

- Thorough peer review

- No space constraints or color figure charges

- Immediate publication on acceptance

- Inclusion in PubMed, CAS, Scopus and Google Scholar

- Research which is freely available for redistribution 